\title{
Publisher's Note: Guided modes of a wire medium slab: Comparison of effective medium approaches with exact calculations [Phys. Rev. B 91, 155427 (2015)]
}

\author{
J. Scott Brownless, Björn C. P. Sturmberg, Alexander Argyros, Boris T. Kuhlmey, and C. Martijn de Sterke \\ (Received 25 March 2016; published 11 April 2016)
}

DOI: 10.1103/PhysRevB.93.159903

This paper was published online on 24 April 2015 with the images of Figs. 5 and 6 transposed. The figures have been corrected as of 28 March 2016. The figures are incorrect in the printed version of the journal, therefore for the benefit of the print readership, the figures and their captions are replicated below.

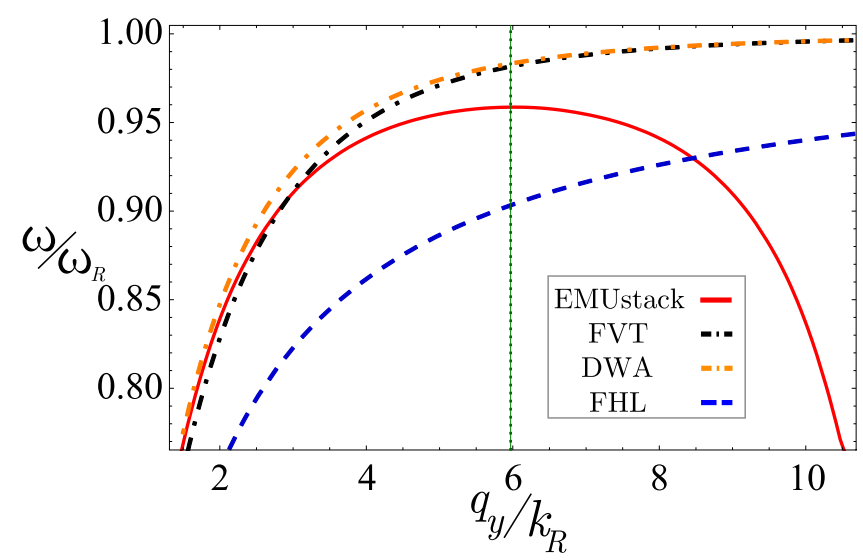

FIG. 5. Dispersion of WM slab modes obtained using different approximations, for $k_{p} d=12$. The blue dashed curve is the full homogeneity limit (FHL), the black dot dashed curve is the full vector treatment (FVT), and the orange curve is the dense wire approximation (DWA). The red curve is simulated results using EMUSTACK with $a \approx \lambda_{R} / 12$. The BZ edge is marked with a green dashed line at $q_{y} \approx 6 k_{R}$.

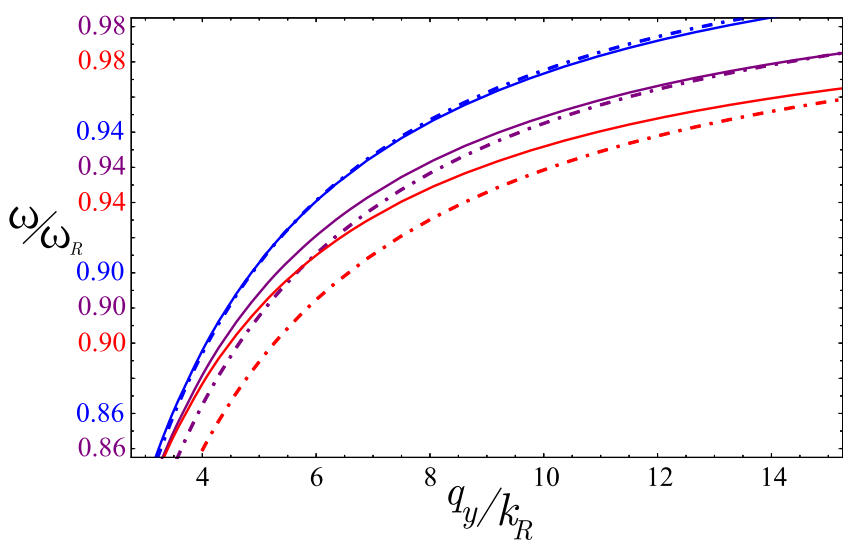

FIG. 6. Comparison of the full vector treatment (dash-dotted curves) and the numerical results from EMUSTACK (solid curves) for three different wire fill fractions, $f_{f}=0.01$ (blue), 0.05 (purple), and 0.1 (red). To avoid confusion with overlapping curves, the three sets have been vertically shifted, where the corresponding frequency values have matching colors. The resonant wavelength of the simulated slab is $\lambda_{R}=0.8$ $\mathrm{m}$, with period $a \approx \lambda_{R} / 100$. 